\title{
The 2016 Young Innovators of Cellular and Molecular Bioengineering
}

It is our pleasure to introduce the 2016 Cellular and Molecular Bioengineering (CMBE) Young Innovators. This is the third year of this competition, and we are excited to see the depth and quality of research in the field of Cellular and Molecular Bioengineering continuing to grow, including new methodologies and approaches that may change the field for years to come.

The corresponding authors in this issue were selected through a competitive process that was initiated in Fall 2015 when self-nominations were solicited from members of the Biomedical Engineering Society (BMES). The competition is open to all researchers holding the rank of assistant professor (or equivalent) working in the field of Cellular and Molecular Bioengineering. Applicants were asked to submit a biosketch and an abstract to be considered for the competition. This year's Young Innovators were chosen from a highly competitive pool of candidates. As a result of the selection process, chosen authors were invited to submit full-length manuscripts that underwent a rigorous peer-review process prior to publication in this invited issue. The application pool was outstanding and as a result, the Young Innovators featured here include many of the best and brightest working in the field.

A group of Young Innovator articles are focused on bioengineering research at the molecular scales. Kathryn Whitehead and colleagues show that the tail length and structure of lipidoid molecules assembled into nanoparticles significantly affect the efficacy of siRNA delivery. Steven Jay and colleagues demonstrate that the HER2 oncogene can be knocked down via small RNAs loaded into extracellular vesicles. Xiaojun Lian et al. examined Wnt signaling in human pluripotent stem cells using CRISPR-Cas9 gene editing. Gregory Hudalla and colleagues present a new approach for the self assembly of biomaterials termed "CATCH", which stands for "co-assembly tags based on charge complementarity" and show that this tool can be used to incorporate folded proteins into fluorescent nanofibers and other functional structures.

At the cellular level, Stacey Finley and colleagues present a predictive computational model of $\mathrm{T}$ cell activation via lymphocyte-specific protein tyrosine kinase autoregulation. Craig Duvall and colleagues show that oppositely charged peptide inhibitor and $\mathrm{pH}$-responsive polymer "nano-polyplexes" are able to sig- nificantly increase cellular uptake and intracellular retention. Through electron microscopy and endocytosis inhibition they found that the uptake involves both macropinocytosis and clathrin-mediated endocytosis, whereas free peptide is internalized solely via the clathrin-mediated mechanism. Minglin $\mathrm{Ma}$ and colleagues describe an interesting new approach that coats individual cells with a drug-eluting mixture of cholesterol and dextran nanoparticles, and demonstrate their technology by delivering therapeutic curcumin to individual cells. In another Young Innovator study led by Kandice Tanner, the mechanical properties of the tumor stromal microenvironment was probed via optical trap-based microrheology, finding nonlinear stress-strain responses and different physical properties at the microscale.

Finally, several of the Young Innovator articles are focused on multicellular phenomena. Christopher Jewell and colleagues used intra-lymph node injection to study the local and systemic responses to microparticle vaccines encapsulating toll-like receptor agonists for melanoma therapy. Erkin Seker and colleagues present nanoporous gold nanotopographies that can maintain high neuronal cell coverage while reducing astrocyte surface coverage for use as neural electrodes. Finally, Ting $\mathrm{Lu}$ and coworkers describe their development of an integrated model of bacteriocin synthesis and quorum sensing in a two-species growth environment, with important implications for microbial ecology.

The 2016 CMBE Young Innovators will present their research at the 2016 Annual Biomedical Engineering Society Meeting in Minneapolis, Minnesota on October 5-8, 2016. A special two-part platform session will be held as part of the Cellular and Molecular Bioengineering track, and each corresponding author will present his or her research and be recognized as a 2016 CMBE Young Innovator. We encourage you to attend and learn more about the exciting and innovative research being conducted by these investigators, and further interact with the journal on Twitter (www.twitter.com/CMBEjournal) and Facebook (www.facebook.com/CMBEjournal) throughout the year.

The 2017 CMBE Young Innovators competition is now underway, with self-nominations due by November 4, 2016. Interested researchers who hold a position at the rank of Assistant Professor (or equivalent) are 
invited to submit a 200-word abstract and a 2-page NIH-style biosketch to Editor-in-Chief Michael King at mike.king@cornell.edu. Note that starting with the 2017 competition, CMBE Young Innovators will no longer be required to be members of the Biomedical Engineering Society, and non-members are eligible and welcome to submit their nominations. Please consider nominating yourself or encouraging your eligible colleagues to do so. We hope that you will enjoy this year's Young Innovators issue, and we look forward to communicating more of the most innovative research in CMBE in 2017 and beyond!
Tejal A. Desai

Department of Bioengineering and Therapeutic Sciences, Schools of Pharmacy and Medicine, University of California at San Francisco,

San Francisco, CA 94158, USA

Michael R. King

Meinig School of Biomedical Engineering, Cornell University, 205 Weill Hall, 526 N. Campus Rd, Ithaca, NY 14853, USA

Electronic mail: mike.king@cornell.edu 